\title{
STATE-DEPENDENT FRACTIONAL POINT PROCESSES
}

\author{
R. GARRA* AND \\ E. ORSINGHER, ${ }^{* *}$ Sapienza Università di Roma \\ F. POLITO, ${ }^{* * *}$ Università degli Studi di Torino
}

\begin{abstract}
In this paper we analyse the fractional Poisson process where the state probabilities $p_{k}^{v_{k}}(t)$, $t \geq 0$, are governed by time-fractional equations of order $0<v_{k} \leq 1$ depending on the number $k$ of events that have occurred up to time $t$. We are able to obtain explicitly the Laplace transform of $p_{k}^{v_{k}}(t)$ and various representations of state probabilities. We show that the Poisson process with intermediate waiting times depending on $v_{k}$ differs from that constructed from the fractional state equations (in the case of $v_{k}=v$, for all $k$, they coincide with the time-fractional Poisson process). We also introduce a different form of fractional state-dependent Poisson process as a weighted sum of homogeneous Poisson processes. Finally, we consider the fractional birth process governed by equations with state-dependent fractionality.
\end{abstract}

Keywords: Dzhrbashyan-Caputo fractional derivative; Poisson process; stable process; Mittag-Leffler function; pure birth process

2010 Mathematics Subject Classification: Primary 60G55; 26A33

Secondary 34A08; 60G22

\section{Introduction}

We first consider a state-dependent time-fractional Poisson process $N(t), t \geq 0$, whose state probabilities $p_{k}^{v_{k}}(t)=\mathbb{P}\{N(t)=k\}$ are governed by the following equations

$$
\begin{gathered}
\frac{\mathrm{d}^{\nu_{k}}}{\mathrm{~d} t^{\nu_{k}}} p_{k}^{\nu_{k}}(t)=-\lambda p_{k}^{\nu_{k}}(t)+\lambda p_{k-1}^{\nu_{k-1}}(t), \quad k \geq 0, t>0, v_{k} \in(0,1], \lambda>0, \\
p_{k}^{v_{k}}(0)= \begin{cases}1, & k=0, \\
0, & k \geq 1,\end{cases}
\end{gathered}
$$

where $p_{k}^{v_{k}}(t)=0$ if $k \in \mathbb{Z}^{-} \backslash\{0\}$. These equations are obtained by replacing, in the governing equations of the homogeneous Poisson process, the ordinary derivative with the DzhrbashyanCaputo fractional derivative (see Podlubny (1999)),

$$
\frac{\mathrm{d}^{v}}{\mathrm{~d} t^{\nu}} f(t)= \begin{cases}\frac{1}{\Gamma(m-v)} \int_{0}^{t}(t-s)^{m-v-1} f^{(m)}(s) \mathrm{d} s, & m-1<v<m, \\ \frac{\mathrm{d}^{m} f}{\mathrm{~d} t^{m}}, & v=m .\end{cases}
$$

Received 12 April 2013; revision received 3 March 2014.

* Postal address: Dipartimento di Scienze di Base e Applicate per l'Ingegneria, Sapienza Università di Roma, Via A.Scarpa 16, 00161, Roma, Italy. Email address: rolinipame@yahoo.it

** Postal address: Dipartimento di Statistica, Sapienza Università di Roma, Piazzale Aldo Moro 5, 00185 Roma, Italy. *** Postal address: Dipartimento di Matematica "G. Peano", Università degli Studi di Torino, Via Carlo Alberto 10, 10123 Torino, Italy. 
We remark that in (1.1), the order of the fractional derivatives depends on the number of events that have occurred up to time $t$. By definition, we have

$$
\frac{\mathrm{d}^{\nu_{k}}}{\mathrm{~d} t^{\nu_{k}}} p_{k}^{v_{k}}(t)=\frac{1}{\Gamma\left(1-v_{k}\right)} \int_{0}^{t}(t-s)^{-v_{k}} \frac{\mathrm{d}}{\mathrm{d} s} p_{k}^{v_{k}}(s) \mathrm{d} s, \quad 0<v_{k}<1 .
$$

Hence, the dependence of $p_{k}^{\nu_{k}}(t)$ on the past is twofold. On one side, the fractional derivative depends on the whole time span $[0, t]$ through the weight function. On the other side, the number of events that have occurred up to time $t$ modifies the power of the weight function. This means that in the case of a monotonical structure of the sequence of fractional orders $v_{k}$, the memory effect can play an increasing or decreasing role. For example, if $v_{k}$ decreases with $k$, the memory function tends to be constant and applies the same weight to the whole time span $[0, t]$. We note that state-dependent fractionality was considered in different contexts by Fedotov et al. (2012).

For $v_{k}=v$, for all $k$, the system (1.1) coincides with the system governing the classical fractional Poisson process considered for example by Beghin and Orsingher (2009), where the fractional derivative is meant in the Dzhrbashyan-Caputo sense as in our case. Of course, if $v_{k}=1$, for all $k$, we retrieve the governing equation for the homogeneous Poisson process. Some papers devoted to the study of various forms of fractional Poisson processes have appeared in the last decades. In Hilfer and Anton (1995), the authors introduced for the first time the MittagLeffler waiting-time density in the theory of continuous-time random walks. The time-fractional Poisson process was then explicitly considered by Repin and Saichev (2000). Starting from this paper, different approaches to fractional Poisson processes were considered. In Mainardi et al. (2004), for example, the authors considered renewal processes with Mittag-Leffler distributed intertimes. A slightly different approach to the fractional Poisson process was developed by Laskin (2003), where the fractional derivative appearing in the equations governing the state probabilities coincides with the Riemann-Liouville derivative. More recently, Beghin and Orsingher (2009) and Meerschaert et al. (2011) studied the subordination of the Poisson process to the inverse stable subordinator, discussing the relation with fractional Poisson processes. Another type of fractional Poisson process was developed in Orsingher and Polito (2012) where a space-fractionality was considered. Physical applications of the fractional Poisson processes are discussed, for example, in Laskin (2009), where a new family of quantum coherent states was studied.

By solving (1.1), we obtain

$$
\int_{0}^{+\infty} \mathrm{e}^{-s t} p_{k}^{\nu_{k}}(t) \mathrm{d} t=\frac{\lambda^{k} s^{\nu_{0}-1}}{\prod_{j=0}^{k}\left(s^{\nu_{j}}+\lambda\right)}, \quad s>0 .
$$

The inversion of (1.2) is by no means a simple matter. We have been able to obtain an explicit result for $p_{0}^{\nu_{0}}$ and $p_{1}^{\nu_{1}}$ in terms of generalized Mittag-Leffler functions defined as (see, for example, Saxena et al. (2006))

$$
E_{\nu, \beta}^{m}(x)=\sum_{k=0}^{\infty} \frac{x^{k} \Gamma(m+k)}{k ! \Gamma(\nu k+\beta) \Gamma(m)}, \quad \nu, \beta, m \in \mathbb{R}^{+}, x \in \mathbb{R} .
$$

We also state the distribution $p_{k}^{v_{k}}(t)$ of the Poisson process with fractionality $v_{k}$ depending on the number of events $k$, in terms of subordinators and their inverses (see (2.12) below). 
Part of this paper is devoted to the construction of a point process $\mathcal{N}(t), t \geq 0$, with intertime $U_{k}$ between the $k$ th and $(k+1)$ th event distributed as

$$
\mathbb{P}\left\{U_{k}>t\right\}=E_{v_{k}, 1}\left(-\lambda t^{\nu_{k}}\right) .
$$

The Laplace transform of the univariate distributions of $\mathcal{N}(t), t \geq 0$, is

$$
\int_{0}^{\infty} \mathrm{e}^{-s t} \mathbb{P}\{\mathcal{N}(t)=k\} \mathrm{d} t=\lambda^{k} \frac{s^{\nu_{k}-1}}{\prod_{j=0}^{k}\left(s^{v_{j}}+\lambda\right)},
$$

which differs slightly from (1.2). From this point of view the state-dependent fractional Poisson process differs from the time-fractional Poisson process because the approach based on the construction by means of independent interevent times $U_{k}$ and the one based on fractional equations (1.1), do not lead to the same one-dimensional distribution. We show that the probabilities $p_{k}(t)=\mathbb{P}\{\mathcal{N}(t)=k\}$ are solutions to the fractional integral equations

$$
p_{k}(t)-p_{k}(0)=-\lambda I^{v_{k}} p_{k}(t)+\lambda I^{v_{k-1}} p_{k-1}(t),
$$

where $I^{v_{k}}$ is the Riemann-Liouville fractional integral

$$
\left(I^{v_{k}} f\right)(t)=\frac{1}{\Gamma\left(v_{k}\right)} \int_{0}^{t}(t-s)^{v_{k}-1} f(s) \mathrm{d} s, \quad v_{k}>0 .
$$

A third definition of the state-dependent fractional Poisson process, say $\hat{N}(t)$, with distribution

$$
\operatorname{Pr}\{\widehat{N}(t)=j\}=\frac{(\lambda t)^{j}}{\Gamma\left(v_{j} j+1\right)} \frac{1}{E_{v_{j}, 1}(\lambda t)}\left[\sum_{j=0}^{+\infty} \frac{(\lambda t)^{j}}{\Gamma\left(v_{j} j+1\right)} \frac{1}{E_{v_{j}, 1}(\lambda t)}\right]^{-1}, \quad j \geq 0,
$$

is introduced and analysed in Section 3. The distribution

$$
\mathbb{P}\left\{\widehat{N}_{v}(t)=j\right\}=\frac{(\lambda t)^{j}}{\Gamma(v j+1)} \frac{1}{E_{v, 1}(\lambda t)},
$$

investigated in Beghin and Orsingher (2009), has been proved to be a weighted sum of Poisson distributions by Balakrishnan and Kozubowski (2008) and Beghin and Macci (2012).

Finally, we analyse the state-dependent nonlinear pure birth process with one initial progenitor, where the state probabilities $p_{k}^{v_{k}}(t)$ satisfy the fractional equations

$$
\begin{gathered}
\frac{\mathrm{d}^{\nu_{k}}}{\mathrm{~d} t^{\nu_{k}}} p_{k}^{\nu_{k}}(t)=-\lambda_{k} p_{k}^{\nu_{k}}(t)+\lambda_{k-1} p_{k-1}^{v_{k-1}}(t), k \geq 1, \quad t>0, v_{k} \in(0,1], \\
p_{k}^{\nu_{k}}(0)= \begin{cases}1, & k=1, \\
0, & k \geq 2 .\end{cases}
\end{gathered}
$$

The Laplace transform of the solution to (1.5) is given by

$$
\int_{0}^{+\infty} \mathrm{e}^{-s t} p_{k}^{v_{k}}(t) \mathrm{d} t=\left(\prod_{j=1}^{k-1} \lambda_{j}\right) \frac{s^{\nu_{1}-1}}{\prod_{j=1}^{k}\left(s^{\nu_{j}}+\lambda_{j}\right)} .
$$

A similar and more general state-dependent fractional birth-death process was recently tackled by Fedotov et al. (2012), where possible applications to chemotaxis are sketched. The case where $v_{k}=v$, for all $k$ in (1.5), has been dealt with in Orsingher and Polito (2010). An attempt 
to apply this fractional birth process was discussed in Garra and Polito (2011) in relation to tumoral growth models and ETAS (epidemic type aftershock sequences) models in statistical seismology. The dependence of the state probabilities of the point processes considered here from the structure of $v_{k}$ requires further investigation which certainly implies a numerical approach.

\subsection{Notation}

For the sake of clarity we briefly summarize the notation used for the different point processes analysed in the following sections. First, we indicate with $N(t), t \geq 0$, the counting process associated with the variable-order difference-differential equations (1.1). In particular, the state probabilities $p_{k}^{v_{k}}(t)=\mathbb{P}\{N(t)=k\}, k \geq 0$, represent the probability of being in state $k$ at a fixed time $t \geq 0$. The point process constructed and studied in Section 3 by means of independent but nonidentically distributed interarrival times is instead indicated by $\mathcal{N}(t)$. Both processes, when $v_{k}=v$ for all $k \geq 0$, reduce to the time-fractional Poisson process $N_{v}(t)$ treated, for example, in Beghin and Orsingher (2009). In the same article the authors also considered the alternative definition for a fractional Poisson process characterized by the distribution (1.4) and denoted here by $\hat{N}_{v}(t), t \geq 0$. We refer to its direct generalization in a state-dependent sense as $\hat{N}(t)$ for which the distribution becomes that in (1.3). Lastly, the linear fractional pure birth process with state-dependent order of fractionality presented in the last section is simply indicated as $N_{\text {lin }}(t), t \geq 0$.

\section{The state-dependent fractional Poisson process}

We first consider a state-dependent time-fractional Poisson process $N(t), t \geq 0$, whose state probabilities $p_{k}^{\nu_{k}}(t)=\mathbb{P}\{N(t)=k\}$ are governed by (1.1). We have the following result.

Theorem 2.1. The Laplace transform of the solution to the state-dependent time-fractional equations

$$
\begin{gathered}
\frac{\mathrm{d}^{v_{k}}}{\mathrm{~d} t^{\nu_{k}}} p_{k}^{\nu_{k}}(t)=-\lambda p_{k}^{\nu_{k}}(t)+\lambda p_{k-1}^{\nu_{k-1}}(t), \quad k \geq 0, t>0, v_{k} \in(0,1], \\
p_{k}^{v_{k}}(0)= \begin{cases}1, & k=0, \\
0, & k \geq 1,\end{cases}
\end{gathered}
$$

can be written as

$$
\tilde{p}_{k}^{\nu_{k}}(s)=\int_{0}^{+\infty} \mathrm{e}^{-s t} p_{k}^{v_{k}}(t) \mathrm{d} t=\frac{\lambda^{k} s^{\nu_{0}-1}}{\prod_{j=0}^{k}\left(s^{\nu_{j}}+\lambda\right)},
$$

where the fractional derivative appearing in (2.1) is in the sense of Dzhrbashyan-Caputo.

Proof. We can solve (2.1) by means of an iterative procedure, as follows. The equation related to $k=0$ is

$$
\frac{\mathrm{d}^{\nu_{0}}}{\mathrm{~d} t^{\nu_{0}}} p_{0}^{\nu_{0}}(t)=-\lambda p_{0}^{\nu_{0}}(t), \quad t>0, \nu_{0} \in(0,1], \quad p_{0}^{\nu_{0}}(0)=1,
$$

and has the solution $p_{0}^{\nu_{0}}(t)=E_{\nu_{0}, 1}\left(-\lambda t^{\nu_{0}}\right)$, with Laplace transform,

$$
\tilde{p}_{0}^{\nu_{0}}(s)=\int_{0}^{+\infty} \mathrm{e}^{-s t} p_{0}^{\nu_{0}}(t) \mathrm{d} t=\frac{s^{\nu_{0}-1}}{\lambda+s^{\nu_{0}}},
$$


where

$$
E_{\nu_{0}, 1}\left(-\lambda t^{\nu_{0}}\right)=\sum_{k=0}^{\infty} \frac{\left(-\lambda t^{\nu_{0}}\right)^{k}}{\Gamma\left(\nu_{0} k+1\right)}
$$

is the Mittag-Leffler function.

For $k=1$,

$$
\left\{\begin{array}{l}
\frac{\mathrm{d}^{\nu_{1}}}{\mathrm{~d} t^{\nu_{1}}} p_{1}^{\nu_{1}}(t)=-\lambda p_{1}^{\nu_{1}}(t)+\lambda p_{0}^{\nu_{0}}(t), \quad t>0, v_{1} \in(0,1] \\
p_{1}^{\nu_{1}}(0)=0
\end{array}\right.
$$

has the solution, with Laplace transform,

$$
\tilde{p}_{1}^{\nu_{1}}(s)=\int_{0}^{+\infty} \mathrm{e}^{-s t} p_{1}^{\nu_{1}}(t) \mathrm{d} t=\frac{\lambda s^{\nu_{0}-1}}{\lambda+s^{\nu_{0}}} \frac{1}{\lambda+s^{\nu_{1}}} .
$$

By iterating this procedure, we arrive at

$$
\tilde{p}_{k}^{v_{k}}(s)=\int_{0}^{+\infty} \mathrm{e}^{-s t} p_{k}^{v_{k}}(t) \mathrm{d} t=\frac{\lambda^{k} s^{\nu_{0}-1}}{\prod_{j=0}^{k}\left(s^{\nu_{j}}+\lambda\right)} .
$$

Remark 2.1. A direct approach based on the inversion of the Laplace transform of (2.3) is clumsy and cumbersome. We give the explicit evaluation of $p_{1}^{\nu_{1}}(t)$. In this case, from (2.2), we have

$$
\begin{aligned}
\tilde{p}_{1}^{\nu_{1}}(s) & =\int_{0}^{+\infty} \mathrm{e}^{-s t} p_{1}^{\nu_{1}}(t) \mathrm{d} t \\
& =\frac{\lambda s^{\nu_{0}-1}}{\lambda^{2}+\lambda\left(s^{\nu_{0}}+s^{\nu_{1}}\right)+s^{\nu_{0}+\nu_{1}}} \\
& =\frac{\lambda s^{\nu_{0}-1}}{\lambda^{2}+s^{\nu_{0}+\nu_{1}}} \frac{1}{1+\lambda\left(s^{\nu_{0}}+s^{\nu_{1}}\right) /\left(\lambda^{2}+s^{\nu_{0}+\nu_{1}}\right)} \\
& =\lambda s^{\nu_{0}-1} \sum_{m=0}^{\infty} \frac{\left(-\lambda\left(s^{\nu_{0}}+s^{\nu_{1}}\right)\right)^{m}}{\left(\lambda^{2}+s^{\nu_{0}+\nu_{1}}\right)^{m+1}} \\
& =\lambda s^{\nu_{0}-1} \sum_{m=0}^{\infty} \frac{(-\lambda)^{m}}{\left(\lambda^{2}+s^{\nu_{0}+\nu_{1}}\right)^{m+1}} \sum_{r=0}^{m}\left(\begin{array}{c}
m \\
r
\end{array}\right) s^{\nu_{0} r+\nu_{1}(m-r)} .
\end{aligned}
$$

The inversion of (2.4) involves the generalized Mittag-Leffler function, defined as (see, for example, Saxena et al. (2006))

$$
E_{\nu, \beta}^{m}\left(-\lambda t^{\nu}\right)=\sum_{k=0}^{\infty} \frac{\left(-\lambda t^{\nu}\right)^{k} \Gamma(m+k)}{k ! \Gamma(\nu k+\beta) \Gamma(m)},
$$

where $v, \beta, m \in \mathbb{R}^{+}$. Indeed, we recall the following relation.

$$
\int_{0}^{+\infty} \mathrm{e}^{-s t} t^{\beta-1} E_{\nu, \beta}^{m}\left(-\lambda t^{\nu}\right) \mathrm{d} t=\frac{s^{v m-\beta}}{\left(\lambda+s^{\nu}\right)^{m}} .
$$


In view of (2.4) and (2.5), we arrive at

$$
p_{1}^{\nu_{1}}(t)=\sum_{m=0}^{\infty}(-1)^{m} \lambda^{m+1} \sum_{r=0}^{m}\left(\begin{array}{c}
m \\
r
\end{array}\right) t^{\nu_{0}(m-r)+\nu_{1} r+\nu_{1}} E_{\nu_{0}+\nu_{1}, \nu_{0}(m-r)+v_{1} r+v_{1}+1}^{m+1}\left(-\lambda^{2} t^{\nu_{0}+v_{1}}\right) .
$$

For the $v_{1}=v_{0}=v$ case, (2.6) becomes

$$
\begin{aligned}
& p_{1}^{v}(t)=\sum_{m=0}^{\infty}(-1)^{m} \lambda^{m+1} \sum_{r=0}^{m}\left(\begin{array}{l}
m \\
r
\end{array}\right) t^{\nu(m+1)} E_{2 v, v(m+1)+1}^{m+1}\left(-\lambda^{2} t^{2 v}\right) \\
& =\sum_{m=0}^{\infty}(-1)^{m}\left(\lambda t^{\nu}\right)^{m+1} 2^{m} E_{2 v, v(m+1)+1}^{m+1}\left(-\lambda^{2} t^{2 v}\right) \\
& =\sum_{m=0}^{\infty}(-1)^{m}\left(\lambda t^{v}\right)^{m+1} 2^{m} \sum_{r=0}^{\infty}\left(\begin{array}{c}
m+r \\
r
\end{array}\right) \frac{(-1)^{r}\left(\lambda^{2} t^{2 v}\right)^{r}}{\Gamma(2 v r+v(m+1)+1)} \\
& =\sum_{m=0}^{\infty}(-1)^{m}\left(\lambda t^{v}\right)^{m+1} 2^{m} \sum_{r=0}^{\infty}\left(\begin{array}{c}
-(m+1) \\
r
\end{array}\right)\left(\lambda^{2} t^{2 v}\right)^{r} \frac{1}{2 \pi \mathrm{i}} \int_{H a} \mathrm{e}^{w} w^{-2 v r-v(m+1)-1} \mathrm{~d} w \\
& =\sum_{m=0}^{\infty}(-1)^{m}\left(\lambda t^{v}\right)^{m+1} 2^{m} \\
& \times \frac{1}{2 \pi \mathrm{i}} \int_{H a} \mathrm{e}^{w} w^{-v(m+1)-1}\left[\sum_{r=0}^{\infty}\left(\begin{array}{c}
-(m+1) \\
r
\end{array}\right)\left(\lambda^{2} t^{2 v} w^{-2 v}\right)^{r}\right] \mathrm{d} w \\
& =\sum_{m=0}^{\infty}(-1)^{m}\left(\lambda t^{\nu}\right)^{m+1} 2^{m} \frac{1}{2 \pi \mathrm{i}} \int_{H a} \mathrm{e}^{w} \frac{w^{-v(m+1)-1}}{\left(\lambda^{2} t^{2 v} w^{-2 v}+1\right)^{m+1}} \mathrm{~d} w \\
& =\frac{1}{2 \pi \mathrm{i}} \int_{H a} \mathrm{e}^{w} \frac{\lambda t^{v} w^{-v-1}}{\lambda^{2} t^{2 v} w^{-2 v}+1}\left[\sum_{m=0}^{\infty}(-1)^{m}\left(\frac{2 w^{-v} \lambda t^{v}}{\lambda^{2} t^{2 v} w^{-2 v}+1}\right)^{m}\right] \mathrm{d} w \\
& =\frac{1}{2 \pi \mathrm{i}} \int_{H a} \frac{\lambda t^{\nu} w^{\nu-1} \mathrm{e}^{w}}{\left(w^{\nu}+\lambda t^{\nu}\right)^{2}} \mathrm{~d} w \\
& =\frac{\lambda t^{v}}{v} E_{v, v}\left(-\lambda t^{\nu}\right) \text {, }
\end{aligned}
$$

where in the last equality we have used the fact that

$$
E_{v, v}(x)=v \frac{\mathrm{d}}{\mathrm{d} x} E_{\nu, 1}(x)=\frac{v}{2 \pi \mathrm{i}} \frac{\mathrm{d}}{\mathrm{d} x} \int_{H a} \frac{\mathrm{e}^{w} w^{\nu-1}}{w^{\nu}-x} \mathrm{~d} w=\frac{v}{2 \pi \mathrm{i}} \int_{H a} \frac{\mathrm{e}^{w} w^{\nu-1}}{\left(w^{\nu}-x\right)^{2}} \mathrm{~d} w,
$$

and applied the contour-integral representation of the reciprocal of the Gamma function

$$
\frac{1}{\Gamma(x)}=\frac{1}{2 \pi \mathrm{i}} \int_{H a} \mathrm{e}^{u} u^{-x} \mathrm{~d} u
$$

where $H a$ stands for the Hankel contour (see Olver et al. (2010, Equation (5.9.2), p. 139)). 
We note that (2.7) gives the result obtained for the time-fractional Poisson process in Beghin and Orsingher (2009) as expected. Moreover, by considering

$$
\int_{0}^{+\infty} \mathrm{e}^{-s t} \frac{\lambda t^{\nu}}{\nu} E_{v, \nu}\left(-\lambda t^{\nu}\right) \mathrm{d} t=\frac{\lambda s^{\nu-1}}{\left(\lambda+s^{\nu}\right)^{2}}
$$

we retrieve, for the case $v=v_{0}=v_{1}$,

$$
p_{1}^{v}(t)=\frac{\lambda t^{v}}{v} E_{v, v}\left(-\lambda t^{\nu}\right)
$$

which is the result obtained for the time-fractional Poisson process (see Beghin and Orsingher (2009, Equation (2.11))).

By applying Equation (34) of Saxena et al. (2006) it is possible to give an explicit expression for $p_{k}^{v_{k}}(t)$, for any $k \geq 2$, in terms of cumbersome sums of generalized Mittag-Leffler functions.

Remark 2.2. An alternative way to obtain a representation of the state probability in the statedependent Poisson process is given by the following integral approach; starting from (2.3), we have

$$
\begin{aligned}
\tilde{p}_{k}^{\nu_{k}}(s) & =\int_{0}^{+\infty} \mathrm{e}^{-s t} p_{k}^{\nu_{k}}(t) \mathrm{d} t \\
& =\lambda^{k} \frac{s^{\nu_{0}-1}}{\prod_{j=0}^{k}\left(s^{\nu_{j}}+\lambda\right)} \\
& =\left(\int_{0}^{\infty} \mathrm{e}^{-\lambda w_{0}} s^{\nu_{0}-1} \mathrm{e}^{-w_{0} s^{\nu_{0}}} \mathrm{~d} w_{0}\right)\left(\prod_{j=1}^{k} \int_{0}^{\infty} \mathrm{e}^{-\lambda w_{j}} \lambda \mathrm{e}^{-w_{j} s^{\nu_{j}}} \mathrm{~d} w_{j}\right) .
\end{aligned}
$$

For the following developments, it is useful to recall that the inverse process of a $v$-stable subordinator $\mathscr{H}^{v}(t), t \geq 0$, namely $\mathcal{L}^{v}(t), t \geq 0$, is such that

$$
\mathbb{P}\left\{\mathcal{L}^{\nu}(t)<x\right\}=\mathbb{P}\left\{\mathscr{H}^{\nu}(x)>t\right\}, \quad x, t \geq 0 .
$$

Hence, the relation between the law $l_{v}(x, t)$ of the process $\mathcal{L}^{v}(t)$ and the law $h_{v}(x, t)$ of the process $\mathscr{H}^{\nu}(t)$ is given by (see, for example, D'Ovidio et al. (2014))

$$
l_{v}(x, t)=\frac{\mathbb{P}\left\{\mathcal{L}^{v}(t) \in \mathrm{d} x\right\}}{\mathrm{d} x}=\frac{\partial}{\partial x} \mathbb{P}\left\{\mathscr{H}^{v}(x)>t\right\}=\frac{\partial}{\partial x} \int_{t}^{\infty} h_{v}(s, x) \mathrm{d} s,
$$

or, otherwise,

$$
\int_{t}^{\infty} \mathbb{P}\left\{\mathscr{H}^{\nu}(x) \in \mathrm{d} w\right\}=\int_{0}^{x} \mathbb{P}\left\{\mathcal{L}^{v}(t) \in \mathrm{d} z\right\} .
$$

Hence, the density of the inverse process $\mathcal{L}^{v}(t)$ is given by

$$
\mathbb{P}\left\{\mathcal{L}^{\nu}(t) \in \mathrm{d} x\right\}=\frac{\partial}{\partial x} \int_{t}^{\infty} \mathbb{P}\left\{\mathscr{H}^{\nu}(x) \in \mathrm{d} w\right\} .
$$


Therefore, the Laplace transform of $l_{v}(x, t)$ is given by

$$
\begin{aligned}
\tilde{l}_{v}(x, s) & =\int_{0}^{\infty} \mathrm{e}^{-s t} l_{v}(x, t) \mathrm{d} t \\
& =\int_{0}^{\infty} \mathrm{e}^{-s t} \frac{\mathrm{d}}{\mathrm{d} x}\left[\int_{t}^{+\infty} \mathbb{P}\left\{\mathscr{H}^{\nu}(x) \in \mathrm{d} w\right\}\right] \mathrm{d} t \\
& =\frac{\mathrm{d}}{\mathrm{d} x} \int_{0}^{\infty} \mathbb{P}\left\{\mathscr{H}^{\nu}(x) \in \mathrm{d} w\right\} \int_{0}^{w} \mathrm{e}^{-s t} \mathrm{~d} t \\
& =\frac{1}{s} \frac{\mathrm{d}}{\mathrm{d} x}\left[\int_{0}^{\infty}\left(1-\mathrm{e}^{-s w}\right) \mathbb{P}\left\{\mathscr{H}^{\nu}(x) \in \mathrm{d} w\right\}\right] \\
& =s^{\nu-1} \mathrm{e}^{-x s^{\nu}},
\end{aligned}
$$

where we used the fact that

$$
\tilde{h}_{v}(x, s)=\int_{0}^{+\infty} \mathrm{e}^{-s t} h_{v}(x, t) \mathrm{d} t=\mathrm{e}^{-x s^{\nu}} .
$$

We also note that the explicit form of the law of the inverse of the stable subordinator is known in terms of Wright functions (D'Ovidio et al. (2014)). Going back to (2.8), and in view of (2.10), we can write

$$
\begin{aligned}
\tilde{p}_{k}^{\nu_{k}}(s)= & \left(\int_{0}^{\infty} \mathrm{e}^{-\lambda w_{0}} \mathrm{~d} w_{0} \int_{0}^{\infty} \mathrm{e}^{-s t} l_{\nu_{0}}\left(w_{0}, t\right) \mathrm{d} t\right) \\
& \times\left(\prod_{j=1}^{k} \lambda \int_{0}^{\infty} \mathrm{e}^{-\lambda w_{j}} \mathrm{~d} w_{j} \int_{0}^{\infty} \mathrm{e}^{-s x} h_{\nu_{j}}\left(x, w_{j}\right) \mathrm{d} x\right) \\
= & \int_{0}^{\infty} \mathrm{d} w_{0} \mathrm{e}^{-\lambda w_{0}} \ldots \int_{0}^{\infty} \mathrm{d} w_{k} \mathrm{e}^{-\lambda w_{k}} \\
& \times\left[\int_{0}^{\infty} \mathrm{e}^{-s t} l_{v_{0}}\left(w_{0}, t\right) \mathrm{d} t \prod_{j=1}^{k} \lambda \int_{0}^{\infty} \mathrm{e}^{-s x} h_{v_{j}}\left(x, w_{j}\right) \mathrm{d} x\right] .
\end{aligned}
$$

Hence, by inverting the Laplace transform we obtain

$$
\begin{aligned}
p_{k}^{\nu_{k}}(t)=\lambda^{k} \int_{0}^{\infty} & \mathrm{d} w_{0} \mathrm{e}^{-\lambda w_{0}} \int_{0}^{\infty} \mathrm{d} w_{1} \mathrm{e}^{-\lambda w_{1}} \ldots \\
& \times \int_{0}^{\infty} \mathrm{d} w_{k} \mathrm{e}^{-\lambda w_{k}}\left[l_{v_{0}}(w, t) * h_{v_{1}, \ldots, v_{k}}\left(w_{1}, \ldots, w_{k}, t\right)\right]
\end{aligned}
$$

where the symbol ' $*$ ' stands for the convolution of the law of the inverse stable subordinator $l_{v_{0}}$ and the distribution of the sum of $k$ independent stable subordinators $h_{v_{1}, \ldots, v_{k}}\left(w_{1}, \ldots, w_{k}, t\right)$. In other words, $l_{v_{0}}(w, t) * h_{v_{1}, \ldots, v_{k}}\left(w_{1}, \ldots, w_{k}, t\right)$ is the distribution of the random variable

$$
\mathcal{L}^{\nu_{0}}(t)+\sum_{j=1}^{k} \mathscr{H}^{\nu_{j}}(t) .
$$


Remark 2.3. Another interesting characterization of the state-probabilities of the above process is given by the following observation. First of all, since, for $m=1, E_{v, \beta}^{1}(\cdot)=E_{v, \beta}(\cdot)$, from (2.5) we have

$$
\begin{gathered}
\int_{0}^{+\infty} \mathrm{e}^{-s t} t^{\nu-1} E_{\nu, \nu}\left(-\lambda t^{\nu}\right) \mathrm{d} t=\frac{1}{\lambda+s^{\nu}}, \\
\int_{0}^{+\infty} \mathrm{e}^{-s t} E_{\nu, 1}\left(-\lambda t^{\nu}\right) \mathrm{d} t=\frac{s^{\nu-1}}{\lambda+s^{\nu}}
\end{gathered}
$$

Hence, from (2.3), we find that

$$
\begin{aligned}
\tilde{p}_{k}^{\nu_{k}}(s) & =\frac{\lambda^{k} s^{\nu_{0}-1}}{\prod_{j=0}^{k}\left(s^{\nu_{j}}+\lambda\right)} \\
& =\left[\int_{0}^{+\infty} \mathrm{e}^{-s t} E_{\nu_{0}, 1}\left(-\lambda t^{\nu}\right) \mathrm{d} t\right] \prod_{j=1}^{k}\left[\int_{0}^{+\infty} \mathrm{e}^{-s t} \lambda t^{\nu_{j}-1} E_{\nu_{j}, v_{j}}\left(-\lambda t^{\nu_{j}}\right) \mathrm{d} t\right] .
\end{aligned}
$$

On the other hand, from (2.11), we have

$$
\begin{aligned}
\tilde{p}_{k}^{\nu_{k}}(s)= & \left(\int_{0}^{\infty} \mathrm{e}^{-\lambda w_{0}} \mathrm{~d} w_{0} \int_{0}^{\infty} \mathrm{e}^{-s t} l_{\nu_{0}}\left(w_{0}, t\right) \mathrm{d} t\right) \\
& \times\left(\prod_{j=1}^{k} \lambda \int_{0}^{\infty} \mathrm{e}^{-\lambda w_{j}} \mathrm{~d} w_{j} \int_{0}^{\infty} \mathrm{e}^{-s x} h_{\nu_{j}}\left(x, w_{j}\right) \mathrm{d} x\right) \\
= & \left(\int_{0}^{\infty} \mathrm{e}^{-s t} \tilde{l}_{\nu_{0}}(\lambda, t) \mathrm{d} t\right)\left(\prod_{j=1}^{k} \lambda \int_{0}^{\infty} \mathrm{e}^{-s x} \tilde{h}_{v_{j}}(x, \lambda) \mathrm{d} x\right),
\end{aligned}
$$

which clearly coincides with (2.14).

By inverting the Laplace transform, we obtain the following result

$$
p_{k}^{\nu_{k}}(t)=E_{\nu_{0}, 1}\left(-\lambda t^{\nu_{0}}\right) \underset{j=1}{*} \lambda t^{\nu_{j}-1} E_{v_{j}, \nu_{j}}\left(-\lambda t^{\nu_{j}}\right)=\int_{0}^{\infty} E_{v_{0}, 1}\left(-\lambda(t-s)^{\nu_{0}}\right) g(s) \mathrm{d} s,
$$

where $g(s)$ is the $k$ th time iterated convolution of the functions

$$
h_{j}(t)=\lambda t^{v_{j}-1} E_{v_{j}, v_{j}}\left(-\lambda t^{\nu_{j}}\right) .
$$

We note that the last equation can be written in terms of the Prabhakar operator, that is, an integral operator involving a Mittag-Leffler function as kernel (Prabhakar (1971)). From (2.16) we have an integral representation, in explicit form given by

$$
\begin{aligned}
p_{1}^{\nu_{1}}(t)= & \int_{0}^{t} E_{\nu_{0}, 1}\left(-\lambda(t-s)^{\nu_{0}}\right) E_{\nu_{1}, \nu_{1}}\left(-\lambda s^{\nu_{1}}\right) s^{\nu_{1}-1} \mathrm{~d} s \\
p_{2}^{\nu_{2}}(t)= & \int_{0}^{t} \mathrm{~d} s_{1} E_{\nu_{0}, 1}\left(-\lambda\left(t-s_{1}\right)^{\nu_{0}}\right) \\
& \times \int_{0}^{s_{1}} \mathrm{~d} s_{2} s_{2}^{\nu_{1}-1} E_{\nu_{1}, \nu_{1}}\left(-\lambda s_{2}^{\nu_{1}}\right)\left(s_{1}-s_{2}\right)^{\nu_{2}-1} E_{\nu_{2}, \nu_{2}}\left(-\lambda\left(s_{1}-s_{2}\right)^{\nu_{2}}\right)
\end{aligned}
$$




$$
\begin{aligned}
p_{k}^{v_{k}}(t)= & \int_{0}^{t} \mathrm{~d} s_{1} E_{v_{0}, 1}\left(-\lambda\left(t-s_{1}\right)^{\nu_{0}}\right) \int_{0}^{s_{1}} \mathrm{~d} s_{2} \ldots \\
& \times \int_{0}^{s_{k}} \mathrm{~d} s_{k} s_{k}^{\nu_{k-1}-1} E_{\nu_{k-1}, v_{k-1}}\left(-\lambda s_{k}^{\nu_{k-1}}\right)\left(s_{k-1}-s_{k}\right)^{v_{k}-1} E_{v_{k}, v_{k}}\left(-\lambda\left(s_{k-1}-s_{k}\right)^{v_{k}}\right) .
\end{aligned}
$$

In order to find the mean value of the distribution $p_{k}^{v_{k}}(t)$, we multiply all the terms of (2.1) for $k$ and sum over all the states so that

$$
\begin{aligned}
\sum_{k=0}^{\infty} k \frac{\mathrm{d}^{\nu_{k}}}{\mathrm{~d} t^{\nu_{k}}} p_{k}^{\nu_{k}}(t) & =-\lambda \sum_{k=0}^{\infty} k p_{k}^{\nu_{k}}(t)+\lambda \sum_{k=0}^{\infty} k p_{k-1}^{\nu_{k-1}}(t) \\
& =-\lambda \sum_{k=0}^{\infty} k p_{k}^{\nu_{k}}(t)+\lambda \sum_{k=0}^{\infty}(k+1) p_{k}^{\nu_{k}}(t) \\
& =\lambda .
\end{aligned}
$$

In the $v_{k}=v$ case, for all $k$, we have

$$
\frac{\mathrm{d}^{\nu}}{\mathrm{d} t^{\nu}} \sum_{k=0}^{\infty} k p_{k}^{v}(t)=\frac{\mathrm{d}^{\nu}}{\mathrm{d} t^{\nu}} \mathbb{E}\left(N_{\nu}(t)\right)=\lambda,
$$

the solution of which is given by $\mathbb{E}\left(N_{v}(t)\right)=\lambda t^{v} / \Gamma(v+1)$ (see Beghin and Orsingher (2009, Equation (2.7))). We note that it is possible to find an interesting result involving a convergent series by using the Laplace transform in (2.18). Indeed, we have

$$
\sum_{k=0}^{\infty} k s^{v_{k}} \tilde{p}_{k}^{v_{k}}(s)=\lambda s^{-1},
$$

and recalling that

$$
\tilde{p}_{k}^{\nu_{k}}(s)=\frac{\lambda^{k} s^{\nu_{0}-1}}{\prod_{j=0}^{k}\left(s^{\nu_{j}}+\lambda\right)},
$$

we find that

$$
\sum_{k=0}^{\infty} \frac{k \lambda^{k} s^{\nu_{0}+v_{k}}}{\prod_{j=0}^{k}\left(s^{v_{j}}+\lambda\right)}=\lambda .
$$

This result is not trivial and we can check to see that it works, for example, in the special case of $v=v_{k}$ for all $k$, thus,

$$
\begin{aligned}
\sum_{k=1}^{\infty} \frac{k \lambda^{k} s^{2 v}}{\left(s^{v}+\lambda\right)^{k+1}} & =\frac{s^{2 v}}{s^{v}+\lambda} \sum_{k=1}^{\infty} \frac{k \lambda^{k}}{\left(s^{\nu}+\lambda\right)^{k}} \\
& =\frac{\lambda s^{2 v}}{s^{\nu}+\lambda}\left[\frac{\mathrm{d}}{\mathrm{d} w} \sum_{k=1}^{\infty} \frac{w^{k}}{\left(s^{\nu}+\lambda\right)^{k}}\right]_{w=\lambda} \\
& =\frac{\lambda s^{2 v}}{s^{\nu}+\lambda}\left[\frac{\mathrm{d}}{\mathrm{d} w} \frac{w}{s^{\nu}+\lambda-w}\right]_{w=\lambda} \\
& =\frac{\lambda s^{2 v}}{s^{v}+\lambda}\left[\frac{s^{\nu}+\lambda}{\left(s^{v}+\lambda-w\right)^{2}}\right]_{w=\lambda} \\
& =\lambda .
\end{aligned}
$$


Remark 2.4. We note for the probability generating function $G(u, t)$ of the process $N(t)$, $t \geq 0$, the following representation holds for $u \in[0,1]$ :

$$
\begin{aligned}
\int_{0}^{\infty} \mathrm{e}^{-s t} G(u, t) \mathrm{d} t & =\sum_{k=0}^{\infty} u^{k} \int_{0}^{\infty} \mathrm{e}^{-s t} \mathbb{P}\{N(t)=k\} \mathrm{d} t \\
& =\int_{0}^{\infty} \mathrm{e}^{-s t} \mathbb{P}\left\{\min _{\{0 \leq k \leq N(t)\}} X_{k}>1-u\right\} \mathrm{d} t \\
& =\sum_{k=0}^{\infty} \frac{\lambda^{k} u^{k} s^{\nu_{0}-1}}{\prod_{j=0}^{k}\left(\lambda+s^{\nu_{j}}\right)},
\end{aligned}
$$

where $X_{k}, k \geq 1$, are independent and identically distributed random variables uniform in $[0,1]$.

The representation of the probability generating function

$$
G(u, t)=\mathbb{P}\left\{\min _{\{0 \leq k \leq N(t)\}} X_{k}>1-u\right\},
$$

follows the same lines for the time and space fractional Poisson processes described by Orsingher and Polito (2012). In (2.20), the driving process is the state-dependent Poisson process.

\section{Alternative forms of the state-dependent Poisson process}

We now construct a point process with independent but not identically distributed interarrival times. In particular, the waiting time $U_{k}$ between the $k$ th and $(k+1)$ th arrival is distributed with probability distribution function

$$
f_{U_{k}}(t)=\lambda t^{\nu_{k}-1} E_{v_{k}, \nu_{k}}\left(-\lambda t^{\nu_{k}}\right), \quad t>0 .
$$

Let us now call $\mathcal{N}(t), t \geq 0$, such a process which leads us to the following theorem.

Theorem 3.1. The state probabilities $p_{k}(t)$ of the process $\mathcal{N}(t), t \geq 0$, are governed by the integral equation

$$
p_{k}(t)-p_{k}(0)=-\lambda I^{v_{k}} p_{k}(t)+\lambda I^{v_{k-1}} p_{k-1}(t), \quad t \geq 0, v_{k} \in(0,1],
$$

where $I^{v}$ is the fractional integral in the sense of Riemann-Liouville (see (1)). Moreover, their Laplace transforms are given by

$$
\int_{0}^{\infty} \mathrm{e}^{-s t} \mathbb{P}\{\mathcal{N}(t)=k\} \mathrm{d} t=\lambda^{k} \frac{s^{\nu_{k}-1}}{\prod_{j=0}^{k}\left(s^{\nu_{j}}+\lambda\right)} .
$$

Proof. First, we observe that the Laplace transform of the state probabilities can be calculated directly by using the definition of the process $\mathcal{N}(t)$,

$$
\begin{aligned}
\int_{0}^{\infty} & \mathrm{e}^{-s t} \mathbb{P}\{\mathcal{N}(t)=k\} \mathrm{d} t \\
& =\int_{0}^{\infty} \mathrm{e}^{-s t} \mathrm{~d} t\left[\int_{0}^{t} \mathbb{P}\left(U_{0}+\cdots+U_{k-1} \in \mathrm{d} y\right)-\int_{0}^{t} \mathbb{P}\left(U_{0}+\cdots+U_{k} \in \mathrm{d} y\right)\right] \\
& =\frac{1}{s} \int_{0}^{\infty} \mathrm{e}^{-s y}\left[\mathbb{P}\left(U_{0}+\cdots+U_{k-1} \in \mathrm{d} y\right)-\mathbb{P}\left(U_{0}+\cdots+U_{k} \in \mathrm{d} y\right)\right]
\end{aligned}
$$




$$
\begin{aligned}
& =\frac{1}{s}\left[\frac{\lambda^{k}}{\prod_{j=0}^{k-1}\left(\lambda+s^{\nu_{j}}\right)}-\frac{\lambda^{k+1}}{\prod_{j=0}^{k}\left(\lambda+s^{\nu_{j}}\right)}\right] \\
& =\frac{1}{s} \frac{\lambda^{k}\left(\lambda+s^{\nu_{j}}\right)-\lambda^{k+1}}{\prod_{j=0}^{k}\left(\lambda+s^{\nu_{k}}\right)} \\
& =\lambda^{k} \frac{s^{\nu_{k}-1}}{\prod_{j=0}^{k}\left(s^{\nu_{j}}+\lambda\right)} .
\end{aligned}
$$

We note that, unfortunately, it does not coincide with (2.3). Hence, we have two distinct processes that can be matched only by assuming that $v_{k}=v$ for each $k=0,1 \ldots$ (in other words, in the time-fractional Poisson case).

We can also find, in an explicit way, the integral equation governing the probabilities $p_{k}(t)=$ $\mathbb{P}\{\mathcal{N}(t)=k\}$. We start from the ordinary difference-differential equation, governing the Poisson process

$$
\frac{\mathrm{d} p_{k}}{\mathrm{~d} t}(t)=-\lambda p_{k}(t)+\lambda p_{k-1}(t)
$$

with initial conditions

$$
p_{k}(0)= \begin{cases}1, & k=0 \\ 0, & k \geq 1\end{cases}
$$

By integration with respect to $t$, we have the equivalent integral equation

$$
p_{k}(t)-p_{k}(0)=-\lambda \int_{0}^{t} p_{k}(s) \mathrm{d} s+\lambda \int_{0}^{t} p_{k-1}(s) \mathrm{d} s .
$$

In order to obtain a fractional generalization of the last equation, we replace the first-order integral in the right-hand side of (3.2), with state-dependent fractional integrals, i.e.

$$
p_{k}(t)-p_{k}(0)=-\lambda I^{v_{k}} p_{k}(t)+\lambda I^{v_{k-1}} p_{k-1}(t), \quad t \geq 0, v_{k} \in(0,1], k \geq 0,
$$

where $I^{v_{k}}$ is the fractional integral in the sense of Riemann-Liouville. For $k=0$, we have

$$
p_{0}(t)-1=-\lambda I^{\nu_{0}} p_{0}(t)
$$

whose solution is given simply by $p_{0}(t)=E_{v_{0}, 1}\left(-\lambda t^{\nu_{0}}\right)$. With $k=1$, we obtain

$$
p_{1}(t)=-\lambda I^{\nu_{1}} p_{1}(t)+\lambda I^{\nu_{0}} p_{0}(t),
$$

whose Laplace transform, after some simple calculations, is given by

$$
\tilde{p}_{1}(t)=\frac{\lambda s^{\nu_{1}-1}}{\left(s^{\nu_{0}}+\lambda\right)\left(s^{\nu_{1}}+\lambda\right)},
$$

and coincides with (3.1) in the $k=1$ case. Then, it is immediate to prove that, for any order $k \geq 1$, the Laplace transform of $p_{k}(t)$, is given by (3.1). This proves that (3.3) is the governing equation for $\mathcal{N}(t), t \geq 0$, as claimed. 
In order to highlight the relation between the two processes $N(t), t \geq 0$, and $\mathcal{N}(t), t \geq 0$, we can write, by rearranging (2.19),

$$
\tilde{p}_{k}^{v_{k}}(s)=s^{\nu_{0}-v_{k}} \frac{\lambda^{k} s^{\nu_{k}-1}}{\prod_{j=0}^{k}\left(s^{\nu_{j}}+\lambda\right)} .
$$

Therefore, if $\left(v_{k}-v_{0}\right)>0$, for fixed $k$, we have

$p_{k}^{v_{k}}(t)=\frac{1}{\Gamma\left(v_{k}-v_{0}\right)} \int_{0}^{t}(t-y)^{\left(v_{k}-v_{0}\right)-1} \mathbb{P}\{\mathcal{N}(y)=k\} \mathrm{d} y=I^{v_{k}-v_{0}} \mathbb{P}\{\mathcal{N}(t)=k\}, \quad t \geq 0$,

where $I^{v_{k}-v_{0}}$ is the Riemann-Liouville fractional integral. Note that, since the RiemannLiouville fractional derivative (that we indicate here with $D^{\alpha}$ ) is the left-inverse operator to the Riemann-Liouville fractional integral, we also obtain the related relation

$$
D^{v_{k}-v_{0}} p_{k}^{v_{k}}(t)=\mathbb{P}\{\mathcal{N}(t)=k\}, \quad t \geq 0,\left(v_{k}-v_{0}\right)>0 .
$$

Conversely, in view of (3.1), we can write

$$
\int_{0}^{\infty} \mathrm{e}^{-s t} \mathbb{P}\{\mathcal{N}(t)=k\} \mathrm{d} t=s^{\nu_{k}-v_{0}} \frac{\lambda^{k} s^{\nu_{0}-1}}{\prod_{j=0}^{k}\left(s^{\nu_{j}}+\lambda\right)},
$$

and, thus, if $\left(v_{0}-v_{k}\right)>0$, for fixed $k$, we obtain

$$
\mathbb{P}\{\mathcal{N}(t)=k\}=\frac{1}{\Gamma\left(v_{0}-v_{k}\right)} \int_{0}^{t}(t-y)^{\left(\nu_{0}-v_{k}\right)-1} p_{k}^{\nu_{k}}(y) \mathrm{d} y=I^{\nu_{0}-v_{k}} p_{k}^{\nu_{k}}(t), \quad t \geq 0,
$$

and

$$
D^{\nu_{0}-v_{k}} \mathbb{P}\{\mathcal{N}(t)=k\}=p_{k}^{v_{k}}(t), \quad t \geq 0,\left(v_{0}-v_{k}\right)>0 .
$$

Therefore, we have the following relation between the state probabilities of the two processes

$$
p_{k}^{v_{k}}(t)= \begin{cases}I^{v_{k}-v_{0}} \mathbb{P}\{\mathcal{N}(t)=k\}, & v_{k}>v_{0}, \\ D^{v_{0}-v_{k}} \mathbb{P}\{\mathcal{N}(t)=k\}, & v_{k}<v_{0}\end{cases}
$$

In order to deepen the meaning of this relation, we consider, as an example, the relation between $p_{1}^{\nu_{1}}(t)$ and $\mathbb{P}\{\mathcal{N}(t)=1\}$.

By inverting the Laplace transform (3.1), we obtain

$$
\begin{aligned}
\mathbb{P}\{\mathcal{N}(t)=1\}= & \sum_{m=0}^{\infty}(-1)^{m} \lambda^{m+1} \\
& \times \sum_{r=0}^{m}\left(\begin{array}{c}
m \\
r
\end{array}\right) t^{\nu_{0}(m-r)+\nu_{1} r+\nu_{0}} E_{\nu_{0}+\nu_{1}, \nu_{0}(m-r)+\nu_{1} r+\nu_{0}+1}^{m+1}\left(-\lambda^{2} t^{\nu_{0}+\nu_{1}}\right),
\end{aligned}
$$

by calculation similar to those given above for $p_{1}^{\nu_{1}}(t)$. Recalling that (Mathai and Haubold (2008, p. 123))

$$
I^{\alpha}\left[t^{\gamma-1} E_{\beta, \gamma}^{m}\left(a t^{\beta}\right)\right]=t^{\alpha+\gamma-1} E_{\beta, \alpha+\gamma}^{m}\left(a t^{\beta}\right),
$$


and assuming that, for example, $v_{1}>v_{0}$, we find that

$$
\begin{aligned}
& I^{\nu_{1}-\nu_{0}} \mathbb{P}\{\mathcal{N}(t)=1\} \\
& =\sum_{m=0}^{\infty}(-1)^{m} \lambda^{m+1} \\
& \times \sum_{r=0}^{m}\left(\begin{array}{c}
m \\
r
\end{array}\right) I^{\nu_{1}-\nu_{0}}\left(t^{\nu_{0}(m-r)+\nu_{1} r+\nu_{0}} E_{\nu_{0}+\nu_{1}, \nu_{0}(m-r)+\nu_{1} r+\nu_{0}+1}^{m+1}\left(-\lambda^{2} t^{\nu_{0}+\nu_{1}}\right)\right) \\
& =\sum_{m=0}^{\infty}(-1)^{m} \lambda^{m+1} \\
& \times \sum_{r=0}^{m}\left(\begin{array}{c}
m \\
r
\end{array}\right) t^{\nu_{0}(m-r)+v_{1} r+v_{1}} E_{\nu_{0}+\nu_{1}, \nu_{0}(m-r)+v_{1} r+\nu_{1}+1}^{m+1}\left(-\lambda^{2} t^{\nu_{0}+\nu_{1}}\right) \\
& =p_{1}^{\nu_{1}}(t) \text {, }
\end{aligned}
$$

as expected.

Moreover, we observe that, since $p_{0}^{\nu_{0}}(t)=\mathbb{P}\{\mathcal{N}(t)=0\}=E_{\nu_{0}, 1}\left(-\lambda t^{\nu_{0}}\right)$, we have

$$
\sum_{k=1}^{\infty} p_{k}^{\nu_{k}}(t)=\sum_{k=1}^{\infty} \mathbb{P}\{\mathcal{N}(t)=k\}=1-E_{\nu_{0}, 1}\left(-\lambda t^{\nu_{0}}\right)
$$

In view of (3.4), this implies that

$$
\begin{aligned}
\sum_{k=1}^{\infty} \mathbb{P}\{\mathcal{N}(t)=k\} & =\sum_{k=1}^{\infty} p_{k}^{v_{k}}(t) \\
& =\sum_{k: v_{k}>v_{0}} I^{v_{k}-v_{0}} \mathbb{P}\{\mathcal{N}(t)=k\}+\sum_{k: v_{k}<v_{0}} D^{\nu_{0}-v_{k}} \mathbb{P}\{\mathcal{N}(t)=k\}
\end{aligned}
$$

The second process we construct, denoted by $\hat{N}(t), t \geq 0$, is given by the following generalization of the Poisson process, whose univariate probabilities are given by

$$
\operatorname{Pr}\{\widehat{N}(t)=j\}=\frac{(\lambda t)^{j}}{\Gamma\left(v_{j} j+1\right)} \frac{1}{E_{v_{j}, 1}(\lambda t)}\left[\sum_{j=0}^{+\infty} \frac{(\lambda t)^{j}}{\Gamma\left(v_{j} j+1\right)} \frac{1}{E_{v_{j}, 1}(\lambda t)}\right]^{-1}, \quad j \geq 0,
$$

where $\lambda>0,0<v_{j} \leq 1$. We can treat it as a generalized Poisson process with state-dependent probabilities. Indeed, we note that, if $v_{j}=1$, for all $j$, we have

$$
\operatorname{Pr}\{\widehat{N}(t)=j\}=\frac{(\lambda t)^{j}}{\Gamma(j+1)} \frac{1}{\mathrm{e}^{\lambda t}}\left[\sum_{j=0}^{+\infty} \frac{(\lambda t)^{j}}{\Gamma(j+1)} \frac{1}{\mathrm{e}^{\lambda t}}\right]^{-1}=\frac{(\lambda t)^{j}}{j !} \mathrm{e}^{-\lambda t}=\operatorname{Pr}\{\mathrm{N}(t)=j\},
$$

which is the state probability of the homogeneous Poisson process. 
A similar construction was adopted by Beghin and Orsingher (2009). We note that an analogous generalization was used by Sixdeniers et al. (1999) in quantum mechanics, in relation to Mittag-Leffler-type coherent states. We now recall from Balakrishnan and Kozubowski (2008) that the distribution (3.5) can be regarded as a weighted Poisson sum. Indeed, we note that

$$
\frac{(\lambda t)^{j}}{\Gamma\left(v_{j} j+1\right)} \frac{1}{E_{v_{j}, 1}(\lambda t)}=\frac{j !}{\Gamma\left(v_{j} j+1\right)} \operatorname{Pr}\{\mathrm{N}(t)=j\}\left[\sum_{k=0}^{+\infty} \frac{k !}{\Gamma\left(v_{j} k+1\right)} \operatorname{Pr}\{\mathrm{N}(t)=k\}\right]^{-1} .
$$

Hence, we have

$$
\begin{aligned}
\mathbb{P}\{\hat{N}(t)=j\}= & {\left[\frac{\left(j ! / \Gamma\left(v_{j} j+1\right)\right) \mathbb{P}\{\mathrm{N}(t)=j\}}{\sum_{k=0}^{+\infty}\left(k ! / \Gamma\left(v_{j} k+1\right)\right) \mathbb{P}\{\mathrm{N}(t)=k\}}\right] } \\
& \times\left[\sum_{j=0}^{+\infty} \frac{\left(j ! / \Gamma\left(v_{j} j+1\right)\right) \mathbb{P}\{\mathrm{N}(t)=j\}}{\sum_{k=0}^{+\infty}\left(k ! / \Gamma\left(v_{j} k+1\right)\right) \mathbb{P}\{\mathrm{N}(t)=k\}}\right]^{-1} .
\end{aligned}
$$

The probability generating function of (3.5) is given by

$$
\begin{aligned}
G(u, t) & =\sum_{k=0}^{\infty} u^{k} \operatorname{Pr}\{\widehat{N}(t)=k\} \\
& =\left[\sum_{k=0}^{+\infty} \frac{(\lambda u t)^{k}}{\Gamma\left(v_{k} k+1\right)} \frac{1}{E_{v_{k}, 1}(\lambda t)}\right]\left[\sum_{k=0}^{+\infty} \frac{(\lambda t)^{k}}{\Gamma\left(v_{k} k+1\right)} \frac{1}{E_{v_{k}, 1}(\lambda t)}\right]^{-1} .
\end{aligned}
$$

In the $v_{j}=v$ case, for all $j \geq 0$, we have

$$
G(u, t)=\left[\sum_{k=0}^{+\infty} \frac{(\lambda u t)^{k}}{\Gamma(\nu k+1)} \frac{1}{E_{v, 1}(\lambda t)}\right]\left[\sum_{k=0}^{+\infty} \frac{(\lambda t)^{k}}{\Gamma(v k+1)} \frac{1}{E_{v, 1}(\lambda t)}\right]^{-1}=\frac{E_{v, 1}(u \lambda t)}{E_{v, 1}(\lambda t)},
$$

that coincides with Equation (4.4) of Beghin and Orsingher (2009).

By means of the generating function we can also find the explicit form of the mean value of the distribution (3.5), i.e.

$$
\begin{aligned}
\mathbb{E} \widehat{N}(t) & =\left[\lambda t \sum_{k=0}^{+\infty} \frac{k(\lambda t)^{k-1}}{\Gamma\left(v_{k} k+1\right)} \frac{1}{E_{v_{k}, 1}(\lambda t)}\right]\left[\sum_{k=0}^{+\infty} \frac{(\lambda t)^{k}}{\Gamma\left(v_{k} k+1\right)} \frac{1}{E_{v_{k}, 1}(\lambda t)}\right]^{-1} \\
& =\left[\lambda t \sum_{k=0}^{+\infty} \frac{(\lambda t)^{k}}{v_{k+1} \Gamma\left(v_{k+1} k+v_{k+1}\right)} \frac{1}{E_{v_{k+1}, 1}(\lambda t)}\right]\left[\sum_{k=0}^{+\infty} \frac{(\lambda t)^{k}}{\Gamma\left(v_{k} k+1\right)} \frac{1}{E_{v_{k}, 1}(\lambda t)}\right]^{-1},
\end{aligned}
$$

such that, when $v_{k}=v$ for all $k$, we recover the case considered in Beghin and Orsingher (2009) and in Beghin and Macci (2012), i.e.

$$
\mathbb{E} \hat{N}_{v}(t)=\frac{\lambda t E_{v, v}(\lambda t)}{v E_{v, 1}(\lambda t)}
$$

We now consider a sequence of a random number of nonnegative independent and identically distributed random variables with distribution $F(\beta)=\mathbb{P}\left(X_{i} \leq \beta\right), i \geq 1$ and represented 
by $\hat{N}(t)$. The distribution of the maximum and minimum of this sequence is given by

$$
\begin{gathered}
\operatorname{Pr}\left\{\max \left(X_{1}, \cdots, X_{\widehat{N}(t)}\right)<\beta\right\} \\
=\left[\sum_{k=0}^{+\infty} \frac{(\lambda F(\beta) t)^{k}}{\Gamma\left(v_{k} k+1\right)} \frac{1}{E_{v_{k}, 1}(\lambda t)}\right]\left[\sum_{k=0}^{+\infty} \frac{(\lambda t)^{k}}{\Gamma\left(v_{k} k+1\right)} \frac{1}{E_{v_{k}, 1}(\lambda t)}\right]^{-1}, \\
\operatorname{Pr}\left\{\min \left(X_{1}, \cdots, X_{\widehat{N}(t)}\right)>\beta\right\} \\
=\left[\sum_{k=0}^{+\infty} \frac{(\lambda[1-F(\beta)] t)^{k}}{\Gamma\left(v_{k} k+1\right)} \frac{1}{E_{v_{k}, 1}(\lambda t)}\right]\left[\sum_{k=0}^{+\infty} \frac{(\lambda t)^{k}}{\Gamma\left(v_{k} k+1\right)} \frac{1}{E_{v_{k}, 1}(\lambda t)}\right]^{-1} .
\end{gathered}
$$

In the $v=v_{k}=1$ case, for all $k$, we recover the distribution of the maximum and minimum of the homogeneous Poisson process.

\section{State-dependent fractional pure birth processes}

In this section we consider a different point process which can be generalized in a statedependent sense as we have done for the fractional Poisson process. Thus, we analyse a state-dependent fractional pure birth process (see Orsingher and Polito (2010) for the fractional case with constant order), where the probabilities are governed by the following equations:

$$
\begin{gathered}
\frac{\mathrm{d}^{\nu_{k}}}{\mathrm{~d} t^{\nu_{k}}} p_{k}^{\nu_{k}}(t)=-\lambda_{k} p_{k}^{\nu_{k}}(t)+\lambda_{k-1} p_{k-1}^{\nu_{k-1}}(t), \quad k \geq 1, t>0, v_{k} \in(0,1], \\
p_{k}^{\nu_{k}}(0)= \begin{cases}1, & k=1, \\
0, & k \geq 2 .\end{cases}
\end{gathered}
$$

As in Section 2 the Laplace transform of the solution to (4.1) can be found rather easily. This is done in the following proposition.

Proposition 4.1. The Laplace transform of the solution to the state-dependent fractional purebirth process (4.1) is given by

$$
\tilde{p}_{k}^{\nu_{k}}(s)=\int_{0}^{+\infty} \mathrm{e}^{-s t} p_{k}^{\nu_{k}}(t) \mathrm{d} t=\left(\prod_{j=1}^{k-1} \lambda_{j}\right) \frac{s^{\nu_{1}-1}}{\prod_{j=1}^{k}\left(s^{\nu_{j}}+\lambda_{j}\right)},
$$

where the fractional derivative appearing in (4.1) is in the sense of Dzhrbashyan-Caputo.

Proof. We can solve equation (4.1) by means of an iterative procedure, as follows. The equation related to $k=1$ is given by

$$
\left\{\begin{array}{l}
\frac{\mathrm{d}^{\nu_{1}}}{\mathrm{~d} t^{\nu_{1}}} p_{1}^{\nu_{1}}(t)=-\lambda_{1} p_{1}^{\nu_{1}}(t), \quad t>0, \nu_{1} \in(0,1], \\
p_{1}^{\nu_{1}}(0)=1
\end{array}\right.
$$

and has solution $p_{1}^{\nu_{1}}(t)=E_{\nu_{1}, 1}\left(-\lambda t^{\nu_{1}}\right)$. For $k=2$,

$$
\left\{\begin{array}{l}
\frac{\mathrm{d}^{\nu_{2}}}{\mathrm{~d} t^{\nu_{2}}} p_{2}^{\nu_{2}}(t)=-\lambda_{2} p_{2}^{\nu_{2}}(t)+\lambda_{1} p_{1}^{\nu_{1}}(t), \quad t>0, \nu_{2} \in(0,1], \\
p_{2}^{\nu_{2}}(0)=0
\end{array}\right.
$$


has solution, with Laplace transform,

$$
\tilde{p}_{2}^{\nu_{2}}(s)=\int_{0}^{+\infty} \mathrm{e}^{-s t} p_{2}^{\nu_{2}}(t) \mathrm{d} t=\frac{\lambda_{1} s^{\nu_{1}-1}}{\lambda_{1}+s^{\nu_{1}}} \frac{1}{\lambda_{2}+s^{\nu_{2}}},
$$

whose inverse is given by (see (2.4))

$$
\begin{aligned}
p_{2}^{\nu_{2}}(t)= & \sum_{m=0}^{\infty}(-1)^{m} \sum_{r=0}^{m}\left(\begin{array}{c}
m \\
r
\end{array}\right) \lambda_{1}^{r+1} \lambda_{2}^{m-r} t^{\nu_{2}(m-r)+\nu_{1} r+\nu_{2}} \\
& \times E_{v_{1}+\nu_{2}, \nu_{2}(m-r)+\nu_{1} r+\nu_{2}+1}^{m+1}\left(-\lambda_{1} \lambda_{2} t^{\nu_{1}+\nu_{2}}\right) .
\end{aligned}
$$

By iterating this procedure, we immediately arrive at

$$
\tilde{p}_{k}^{\nu_{k}}(s)=\int_{0}^{+\infty} \mathrm{e}^{-s t} p_{k}^{\nu_{k}}(t) \mathrm{d} t=\left(\prod_{j=1}^{k-1} \lambda_{j}\right) \frac{s^{\nu_{1}-1}}{\prod_{j=1}^{k}\left(s^{\nu_{j}}+\lambda_{j}\right)},
$$

as claimed.

By recalling (2.13), we obtain the explicit expression of the state probabilities $p_{k}^{v_{k}}(t), k \geq 1$, $t \geq 0$, as

$$
p_{k}^{v_{k}}(t)=E_{v_{1}, 1}\left(-\lambda_{1} t^{\nu_{1}}\right) \underset{j=1}{\stackrel{k}{*}} \lambda_{j} t^{v_{j}-1} E_{v_{j}, \nu_{j}}\left(-\lambda_{j} t^{\nu_{j}}\right)
$$

where the convolution is in the sense of (2.16).

We now consider the state-dependent linear birth process, denoted by $N_{\text {lin }}(t), t \geq 0$. This means that we take $\lambda_{k}=\lambda k$ in (4.1). We have the following theorem.

Theorem 4.1. Let us consider the state-dependent linear birth process $N_{\text {lin }}(t), t \geq 0$, governed by

$$
\begin{gathered}
\frac{\mathrm{d}^{\nu_{k}}}{\mathrm{~d} t^{\nu_{k}}} p_{k}^{\nu_{k}}(t)=-\lambda k p_{k}^{\nu_{k}}(t)+\lambda(k-1) p_{k-1}^{\nu_{k-1}}(t), \quad k \geq 1, t>0, v_{k} \in(0,1], \\
p_{k}^{\nu_{k}}(0)= \begin{cases}1, & k=1, \\
0, & k \geq 2,\end{cases}
\end{gathered}
$$

then the following relation holds:

$$
\sum_{k=1}^{\infty} k^{m} \frac{\mathrm{d}^{\nu_{k}}}{\mathrm{~d} t^{\nu_{k}}} p_{k}^{\nu_{k}}(t)=\lambda \sum_{j=1}^{m-1}\left(\begin{array}{c}
m \\
j
\end{array}\right) \mathbb{E} N_{\text {lin }}^{m-j+1} .
$$

Proof. In order to find explicit relations for the moments of the distribution $N_{\text {lin }}(t)$, we multiply both sides of (4.2) by $k^{m}$ and sum over all the states, obtaining

$$
\begin{aligned}
\sum_{k=1}^{\infty} k^{m} \frac{\mathrm{d}^{\nu_{k}}}{\mathrm{~d} t^{\nu_{k}}} p_{k}^{\nu_{k}}(t) & =-\lambda \sum_{k=1}^{\infty} k^{m+1} p_{k}^{\nu_{k}}(t)+\lambda \sum_{k=1}^{\infty} k^{m}(k-1) p_{k-1}^{v_{k-1}}(t) \\
& =-\lambda \sum_{k=1}^{\infty} k^{m+1} p_{k}^{v_{k}}(t)+\lambda \sum_{k=1}^{\infty} k(k+1)^{m} p_{k}^{v_{k}}(t) \\
& =-\lambda \sum_{k=1}^{\infty} k^{m+1} p_{k}^{v_{k}}(t)+\lambda \sum_{k=1}^{\infty} \sum_{j=0}^{m}\left(\begin{array}{c}
m \\
j
\end{array}\right) k^{m-j+1} p_{k}^{\nu_{k}}(t)
\end{aligned}
$$




$$
\begin{aligned}
& =\lambda \sum_{j=1}^{m}\left(\begin{array}{c}
m \\
j
\end{array}\right) \sum_{k=1}^{\infty} k^{m-j+1} p_{k}^{v_{k}}(t) \\
& =\lambda \sum_{j=1}^{m}\left(\begin{array}{c}
m \\
j
\end{array}\right) \mathbb{E} N_{\mathrm{lin}}^{m-j+1} .
\end{aligned}
$$

Remark 4.1. We can consider in a explicit way the relations involving first and second moments. For example, if we multiply (4.2) for $k$ and sum over all the states, we obtain

$$
\begin{aligned}
\sum_{k=1}^{\infty} k \frac{\mathrm{d}^{\nu_{k}}}{\mathrm{~d} t^{\nu_{k}}} p_{k}^{\nu_{k}}(t) & =-\lambda \sum_{k=1}^{\infty} k^{2} p_{k}^{v_{k}}(t)+\lambda \sum_{k=1}^{\infty} k(k-1) p_{k-1}^{v_{k-1}}(t) \\
& =\lambda \sum_{k=1}^{\infty} k p_{k}^{\nu_{k}}(t) \\
& =\lambda \mathbb{E} N_{\text {lin }}(t) .
\end{aligned}
$$

In the same way, for the second moment, we multiply (4.2) for $k^{2}$, obtaining

$$
\begin{aligned}
\sum_{k=1}^{\infty} k^{2} \frac{\mathrm{d}^{\nu_{k}}}{\mathrm{~d} t^{\nu_{k}}} p_{k}^{\nu_{k}}(t) & =-\lambda \sum_{k=1}^{\infty} k^{3} p_{k}^{v_{k}}(t)+\lambda \sum_{k=1}^{\infty} k^{2}(k-1) p_{k-1}^{\nu_{k-1}}(t) \\
& =\lambda \sum_{k=1}^{\infty} k p_{k}^{\nu_{k}}(t)+2 \lambda \sum_{k=1}^{\infty} k^{2} p_{k}^{\nu_{k}}(t) \\
& =\lambda \mathbb{E} N_{\operatorname{lin}}(t)+2 \lambda \mathbb{E}\left(N_{\operatorname{lin}}\right)^{2}(t) .
\end{aligned}
$$

\section{Acknowledgements}

The authors thank the referee for his/her appreciation of our work and for his/her useful remarks. F. Polito was supported by project AMALFI (Università di Torino/Compagnia di San Paolo).

\section{References}

[1] Balakrishnan, N. and Kozubowski, T. J. (2008). A class of weighted Poisson processes. Statist. Prob. Lett. 78, 2346-2352.

[2] Beghin, L. And Macci, C. (2013). Large deviations for fractional Poisson processes. Statist. Prob. Lett. 83, 1193-1202.

[3] Beghin, L. And Orsingher, E. (2009). Fractional Poisson processes and related planar random motions. Electron. J. Prob. 14, 1790-1827.

[4] D'Ovidio, M., Orsingher, E. And Toaldo, B. (2014). Fractional telegraph-type equations and hyperbolic Brownian motion. Statist. Prob. Lett. 89, 131-137.

[5] Fedotov, S., Ivanov, A. O. And Zubarev, A. Y. (2013). Non-homogeneous random walks, subdiffusive migration of cells and anomolous chemotaxis. Math. Model. Nat. Phenom. 8, 28-43.

[6] Garra, R. and Polito, F. (2011). A note on fractional linear pure birth and pure death processes in epidemic models. Physica A 390, 3704-3709.

[7] Hilfer, R. And Anton, L. (1995). Fractional master equation and fractal time random walks. Phys. Rev. E 51, R848-R851.

[8] Laskin, N. (2003). Fractional Poisson process. Commun. Nonlinear Sci. Numerical Simul. 8, 201-213.

[9] Laskin, N. (2009). Some applications of the fractional Poisson probability distribution. J. Math. Phys. 50, 113513. 
[10] Mainardi, F., Gorenflo, R. and Scalas, E. (2004). A fractional generalization of the Poisson process. Vietnam J. Math. 32, 53-64.

[11] Mathai, A. M. and Haubold, H. J. (2008). Special Functions for Applied Scientists. Springer, New York.

[12] Meerschaert, M. M., Nane, E. and Vellaisamy, P. (2011). The fractional poisson process and the inverse stable subordinator. Electron. J. Prob. 16, 1600-1620.

[13] Olver, F. W. J., Lozier, D. W., Boisvert, R. F. ANd Clark, C. W. (eds) (2010). NIST Handbook of Mathematical Functions. Cambridge University Press.

[14] Orsingher, E. ANd Polito, F. (2010). Fractional pure birth processes. Bernoulli 16, 858-881.

[15] Orsingher, E. And Polito, F. (2012). The space-fractional Poisson process. Statist. Prob. Lett. 82, 852-858.

[16] Podlubny, I. (1999). Fractional Differential Equations. Academic Press, San Diego, CA.

[17] PrabhaKar, T. R. (1971). A singular integral equation with a generalized Mittag-Leffler function in the kernel. Yokohama Math. J. 19, 7-15.

[18] Repin, O. N. ANd SAICHev, A. I. (2000). Fractional Poisson law. Radiophysics Quantum Electron. 43, 738-741.

[19] Saxena, R. K., Mathai, A. M. and Haubold, H. J. (2006). Solutions of fractional reaction-diffusion equations in terms of Mittag-Leffler functions. Internat. J. Scientific Res. 15, 1-17.

[20] Sixdeniers, J.-M., Penson, K. A. And Solomon, A. I. (1999). Mittag-Leffler coherent states. J. Phys. A 32, 7543-7563. 